\title{
Driving the Diffusion of Cesium Atoms of Graphite Monolayers on Metal Surfaces into Rhenium (1010)
}

\author{
A. K. Orujov \\ Baku State University, Baku, Azerbaijan \\ Email: orar@mail.ru
}

Received 22 October 2013; revised 23 November 2013; accepted 24 December 2013

Copyright (C) 2014 by author and Scientific Research Publishing Inc.

This work is licensed under the Creative Commons Attribution International License (CC BY).

http://creativecommons.org/licenses/by/4.0/

cc) (i) Open Access

\section{Abstract}

It has been shown experimentally for the first time that diffusion of cesium $\mathbf{n}$ rhenium takes place at irradiation of atoms flux heated up to $T \leq 1800 \mathrm{~K} \operatorname{Re}(10 \overline{10})$-C. Analysis of thermal desorption spectra showed that cesium forms two high-temperature phases during adsorption of cesium on graphite monolayer in rhenium heated up to $1800 \mathrm{~K}$. The first, intercalated phase starts filling immediately and grows up to maximum concentration. The second, diffusion phase appears with slight delay and grows incessantly during adsorption. It is for the first time that the Fick Equation has been applied for such kind of diffusion. Correspondences $\tau_{\text {dif }}=f(T)$ and average displacement $L=f(T, t)$ have been obtained at diffusion of surface cesium in rhenium in the interval of temperatures $1600 \mathrm{~K}<T<2200 \mathrm{~K}$. At conditions of $v=5 \times 10^{13} \mathrm{~cm}^{-2} \cdot \mathrm{s}^{-1}, U=+2000 \mathrm{~V}, T=1700 \mathrm{~K}$, effectiveness of cesium diffusion $\delta_{\mathrm{Cs}}$ in the system of $\operatorname{Re}(1010)-\mathrm{C}$ was identified with application of suggested methods, which showed $\delta_{\mathrm{cs}}=\mathbf{0 . 4 5}$. Activation energies of Cs atoms diffusion in rhenium from rhenium were found and values $E_{1 p}=5.65 \pm 0.05 \mathrm{eV}$ and $E_{p 1}=5.85 \pm 0.05 \mathrm{eV}$ were obtained respectively. Effect of electrical field on intercalation and cesium diffusion in Re-C was observed.

\section{Keywords}

Surface Ionization, Graphite Monolayer, Intercalation, Diffusion

\section{Introduction}

The discovery of intercalation [1] [2] paved the way for the studying of superefficient diffusion of K, Cs, Na, Ba, Sm and In atoms in metals Ir and Re coated with two-dimensional graphite monolayer. The phenomenon of 
potassium, cesium, samarium, barium and indium atoms diffusion across the graphite monolayer in iridium and potassium in rhenium was discovered and studied during the studies [3]-[7]. It was interesting whether cesium would diffuse through the graphite monolayer in rhenium. For the purposes of studying the cesium atoms diffusion in rhenium, facets of rhenium (1010) had to be obtained, conditions enabling the formation of graphite monolayer on its surface needed to be examined and the conditions of intercalation of the graphite monolayer cesium atoms on the surface of rhenium needed to be studied, the latter of which has been thoroughly investigated in [1] [2]. As shown in the above studies, during the adsorption of Cs on Re - C, intercalated cesium leaves only at extremely high temperatures $T \sim 2200 \mathrm{~K}$ and intercalated cesium prevents the dissolution of graphite islets, communicating to the islets a unique thermal stability, which we think is very questionable. Because a graphite monolayer on the surface of iridium is destroyed and carbon atoms are desorbed at $T \sim 1900 \mathrm{~K}$, while graphite monolayer on the surface of rhenium is degraded and carbon atoms are desorbed at temperatures $\sim 5$ $10 \mathrm{~K}$ above the temperature of obtaining graphite monolayer. In this study, we present only a few cases to understand the sequence of processes of intercalation and diffusion. The purpose of this paper is to investigate the possibility of highly efficient diffusion of Cs atoms in rhenium with a monolayer graphite film.

\section{Experimental Set-Up and Research Methods}

The research was done in a static mass spectrometer of sector type with $90^{\circ}$ inclination angle of the ion trajectories. The mass-spectrometer resolution by peak heights was about 300. The mass-spectrometer has two types of ion sources: 1) source with ionization by electrons (volumetric ionization), 2) source of surface ionization with emitter (surface ionization). The atoms or molecules falling on the emitter surface after ionization by PI are accelerated passing through the collector slit with suppressor grid and enter into mass analyzer. One of the most convenient methods commonly used in the experiment is the method of thermal desorption (TDS) flash. A sample (Figure 1.(1)) was placed in a high vacuum chamber before the entrance slit of mass-spectrometer equipped with device for detecting partial pressure or ion current. Coating an adsorbate on the sample surface can be done in different ways. In this case, it is obtained by adsorption. After obtaining the coating, thermal flash is created by selecting the sample's temperature increase mode. Thus, desorption process takes place, characterized by desorption speed and leading to increase of partial pressure on $\Delta \mathrm{P}$ device or increase of surface ionization (SI) $\mathrm{I}^{+}$ current. As the temperature of sample rises, the rate of particles desorption also increases, which leads to an increase in its instantaneous density. Knowing this increase, surface can be diagnosed, information on the nature

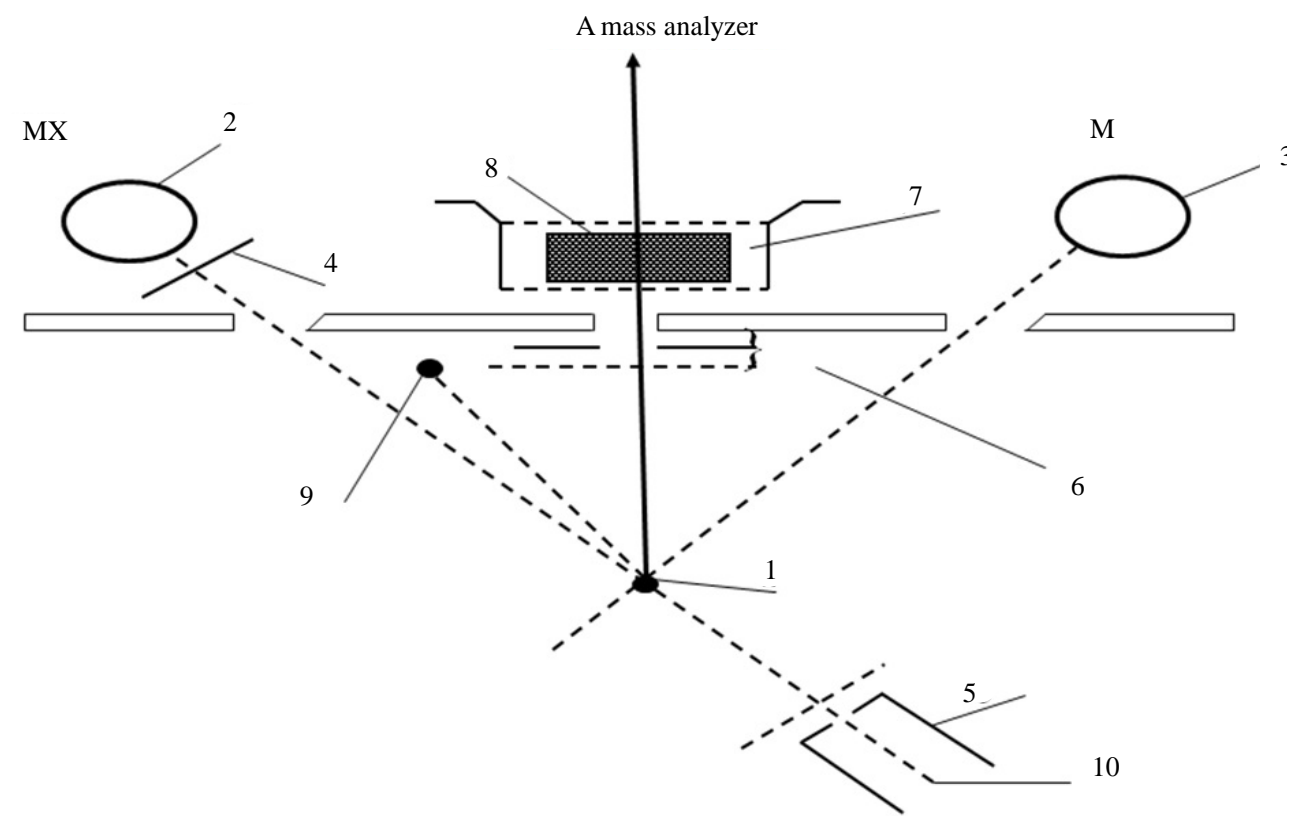

Figure 1. Scheme of ion sources. 1: emitter, rhenium ribbon, 2 - 3: source of molecules or atoms, the Knudsen camera, 4: spoiler, 5: electron gun, 6: manifold with a suppressor grid, 7: box of volume ionization, 8: ions, 9: graphite rod, 10: cathode electron gun. 
and concentration of the adsorbed particles can be obtained and desorption kinetics can be studied. Experiments were performed in ultra-high vacuum with a residual pressure of $10^{-10}$ Torr. Thin strips of $50 \times 1.5 \times 0.03 \mathrm{~mm}^{3}$ textured rhenium with facet (1010) on the surface and work function of $5.1 \mathrm{eV}$ were used. Graphite monolayer was formed by exposing rhenium warmed up to $1600 \mathrm{~K}$ in benzene vapor or spraying from graphite rod (Figure 1.(9)). Surface carbon on rhenium was studied by thermionic emission and dissociation of $\mathrm{CsCl}$ molecules. Graphite monolayer is formed in benzene vapor on rhenium ribbon after saturation of rhenium Re (1010) carbon heated up to $T \leq 1800 \mathrm{~K}$. This is consistent with the results of [8], which are confirmed by Auger electron spectroscopy [1] [2]. Cesium on the surface of $\operatorname{Re}(10 \overline{10})$ - $C$ (with work function $\sim 4.5 \mathrm{eV}$ ) was sprayed from a Knudsen cell, while its state in the adsorbed layer was studied by method of thermal desorption spectroscopy (TDS) in the form of ionic flash with mass-spectrometer registration of Cs ion current that with $\sim 100 \%$ efficiency transformed into cesium $\mathrm{Cs}^{+}$ions during desorption by mechanism of surface ionization, increasing the sensitivity by $\sim 10$ times. To register cesium, the flash was produced at the speed of $-100 \mathrm{deg} / \mathrm{s}$ to $2250 \mathrm{~K}$ rhenium tape temperature and Cs ions current was further recorded on the constant $T$ temperature $\left(T_{\text {пл } \cdot \mathrm{Re}}=3453 \mathrm{~K}\right)$. At such high temperatures, graphite islets in the adsorbed layer of rhenium are destroyed (dissolved), carbon atoms evaporate, while intercalated and diffused cesium is desorbed in the form of $\mathrm{Cs}^{+}$ions. Neutral component of the flow desorbing during the flash was ionized in the source with electron impact before entrance into the mass-analyzer. Figure 2 separately shows the scheme of the volumetric ionizing chamber.

\section{A Brief Theory of the Kinetics of Diffusion between the Surface and Volume of Rhenium}

It is well known that diffusion is an irreversible and non-equilibrium process leading to the establishment of equilibrium distribution of concentrations within phases and Equation of chemical potentials of the system's components. The most important laws of diffusion are the Fick laws [9]. The first law of Fick is like this:

Ion beam
in mass analyzer

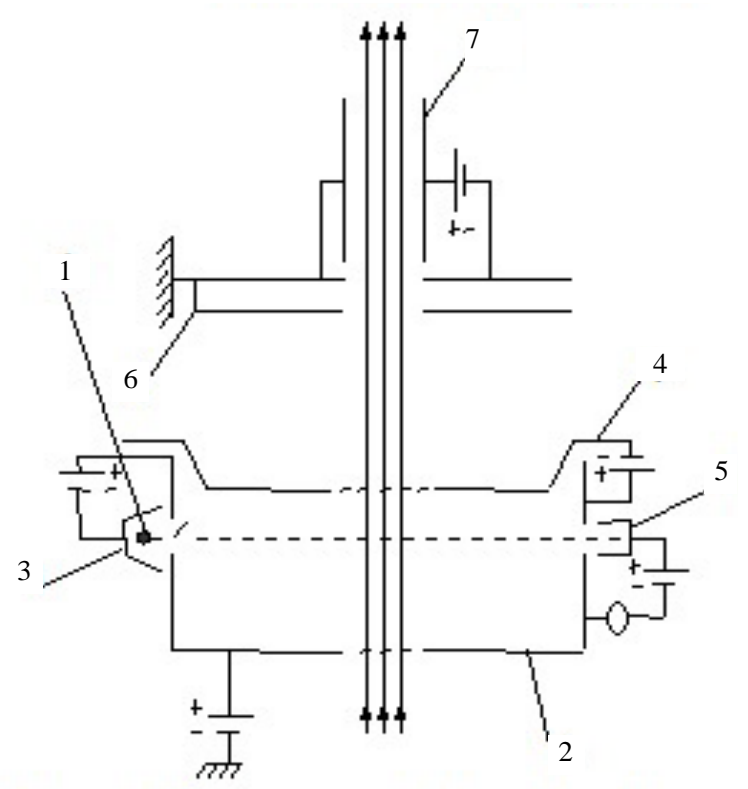

Flow of atoms and molecules

Figure 2. Scheme of ion source with electron ionization (volumetric ionization source). 1: cathode of electron impact, 2: box, 3: focusing electrode, 4: ion extracting electrode, 5: Faraday cup, 6: protective diaphragm, 7: condenser. 


$$
j=-D \nabla n
$$

where $j$ stands for diffusive flux, $D$ —for diffusion coefficient, $\nabla n$ —concentration gradient; the "minus" sign means that the flow is directed towards reducing the concentration;

Fick's second law (for one-dimensional diffusion in the direction of $x$ )

$$
\frac{\partial n}{\partial t}=\frac{\partial}{\partial x}\left(D \frac{\partial n}{\partial x}\right)
$$

In the case of $D$ 's independence on the $n$ concentration, the law looks like this:

$$
\frac{\partial n}{\partial t}=D \frac{\partial^{2} n}{\partial x^{2}}
$$

The diffusion coefficient $D$ shows the number of diffusing substance (in mass units) through unit area under a concentration gradient, equaling to unity; has dimensions $\mathrm{cm}^{2} / \mathrm{sec}$. It is determined by properties of the environment, the type of diffusing particles, depends on the temperature $T$ and the diffusion activation energy $E_{a}$ in accordance with the Arrhenius Equation

$$
D=D_{0} \exp \left(-E_{a} / k T\right)
$$

In this Equation, $D_{0}$ is numerically equal to the diffusion coefficient at the temperature gravitating to infinity. In general, the movement of impurity atoms or ions in the crystal lattice is irregular with overcoming the potential energy barriers. The solution of Equations (2) and (3) has a different appearance depending on the boundary conditions. In particular, boundary conditions for the diffusion from an ongoing source are:

$$
n(0, t)=n_{0} ; \quad n(x, 0)=0
$$

Considering this, the solution is:

$$
\begin{gathered}
n(x, t)=n_{0}\left(1-\operatorname{erf} \frac{x}{2 \sqrt{D t}}\right) \\
\operatorname{erf} z=\frac{2}{\sqrt{\pi}} \int_{0}^{y} \mathrm{e}^{-z^{2}} \mathrm{~d} z
\end{gathered}
$$

where for the Gauss error function (abbreviation erf comes from error function), erfcz = l- erfz complementary error function (error function complement) is used as well.

At the diffusion of adsorbed atoms from intercalated zones with constant surface concentration of metal, the boundary conditions are well observed for our case

$$
n(0, t)=0 ; \quad n(x, 0)=n_{0}
$$

Considering these initial conditions, we obtain an expression for the concentration $n(x, t)$

$$
n(x, t)=n_{0} \operatorname{erf} \frac{x}{2 \sqrt{D t}}
$$

Comparing the literature data for different initial conditions of diffusion, we see that the value of $L=\sqrt{D t}$, which has the dimension of length and is called diffusion length, is included in all the final results of the Fick Equation. The physical meaning of this parameter is the average distance, which the diffusing particles overcome in the direction of alignment of the concentration gradient during the time $t$. The diffusion length is not to be confused with the length of particle's mean free path, which is defined as the average distance traveled by the particle between two successive scattering events. If we assume that atoms diffuse in the form of monovalent positive ions from the intercalated area, then Einstein relationship connecting diffusion coefficient of charged particles with their mobility may be applied in conditions of thermodynamic equilibrium 


$$
\mu=\frac{e D}{k T}
$$

The second Fick Equation taking into account both the diffusion and migration components in the absence of recombination is as follows:

$$
\frac{\partial n}{\partial t}=D \frac{\partial^{2} n}{\partial x^{2}} \pm \mu_{ \pm} E \frac{\partial n}{\partial x}
$$

where the sign "- " refers to the case of positive charges movement (with mobility $\mu_{+}$), and “+”-that of negative charges (mobility $\mu_{-}$), while $E$ stands for the tension of electric field. Various parameters of diffusion can be calculated and assessed by varying the formulas (1)-(11).

\section{Experimental Results and Their Discussion}

It has been shown experimentally that the diffusion of cesium in rhenium occurs at irradiation of atom flows warmed up to $T \leq 1800 \mathrm{~K} \operatorname{Re}(10 \overline{10})$-C. Figure 3 shows the thermal desorption spectra of Cs taken for different times of exposure. An extremely effective diffusion of Cs atoms in $\operatorname{Re}(10 \overline{10})$ has been discovered, stimulated by graphite monolayer on its surface. Analysis of the TD-specters has revealed that similar to the adsorption of $\mathrm{K}$ atoms on the Re-C [8], during adsorption of cesium on monolayer graphite in rhenium heated up to $1800 \mathrm{~K}$, cesium also forms two high-temperature phases. The first intercalated phase starts to be filled immediately and grows up to the maximum concentration. The second diffusion phase appears with some delay and

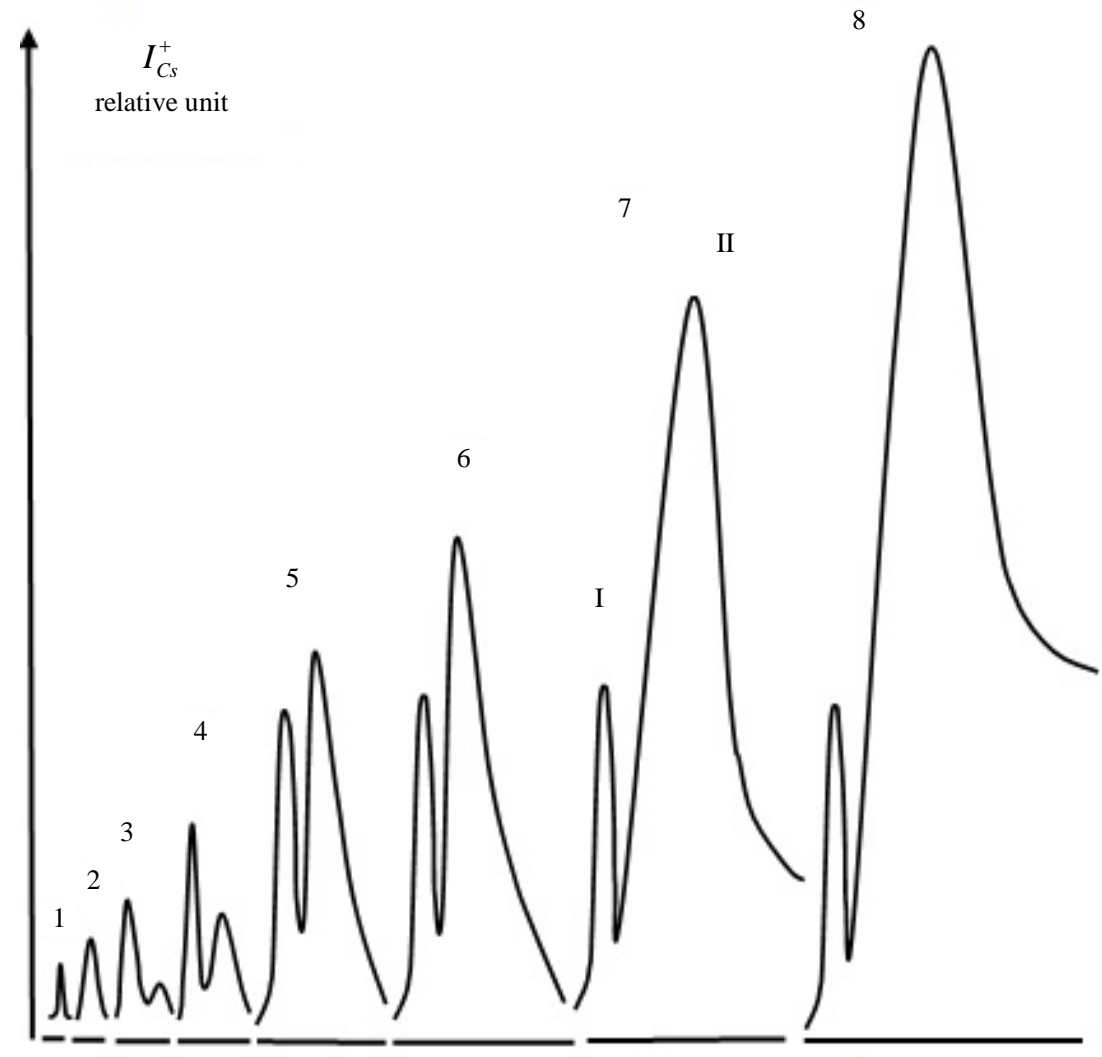

t, time TDC

Figure 3. Thermal desorption spectrum of $\mathrm{Cs}^{+}$ions from graphite monolayer on rhenium which, when heated up to $1800 \mathrm{~K}$, was preliminarily exhibited in the flow of cesium atoms for the duration of time: $t_{e}=1(1), 3(2), 7(3), 12(4), 30(5), 40(6), 60(7), 90$ minutes (8). 
grows continuously during adsorption. The peak's high occupancy, which in some cases exceeded monatomic cesium concentration $\left(\sim 5 \times 10^{14} \mathrm{~cm}^{-2}\right)$ by $10-100$ times on the surface of metals pointed at the diffusive nature of this peak and the thermal removal of cesium required a very long time (tens of hours). Some differences in the TDS of $\mathrm{Cs}^{+}$ions in the systems of $\operatorname{Re}(10 \overline{10})-C$ and $\operatorname{Ir}(111)-C$ were observed. The diffusion phase of rhenium $\mathrm{Cs}^{+}$in comparison with a similar phase of iridium was stretched under the same conditions of preliminary irradiation of $\operatorname{Ir}(111)-\mathrm{C}$, and $\operatorname{Re}(1010)$-C. The above differences in the forms of TDS and effectiveness of cesium atoms diffusion in $\operatorname{Re}(1010)$ and $\operatorname{Ir}(111)$ are apparently related to differences of their crystallographic structures and diffusion lengths. Another difference of cesium TDS peaks from $\operatorname{Ir}(\mathrm{III})-\mathrm{C}$ and $\operatorname{Re}(1010)-\mathrm{C}$ was in the initial desorption temperatures of the diffusion phase. If in the case of $\operatorname{Ir}(\mathrm{III})-\mathrm{C}$, beginning of TDS corresponded with desorption temperature of $T \geq 1900 \mathrm{~K}$ carbon atoms, i.e. temperature of the graphite structure destruction, in the case of $\operatorname{Re}(10 \overline{1} 0)$-C it conformed with delivery temperature of monolayer graphite in Re, i.e. temperature of carbonization.

\section{Average Displacements and Diffusion Length of Cesium Atoms in Rhenium}

Suppose $\tau_{\text {diff }}$ is an average lifetime of the Cs atom in rhenium lattice with respect to the diffusion hopping and $\tau_{\exp }$ is observation time. Then, during the observation time, the Cs atom will make $n=\tau_{\exp } / \tau_{\text {diff }}$ diffusion jumps with chaotic nature of movements, move in the given direction at a $L=\sqrt{D \tau_{\exp }}$ distance or $L=\lambda \sqrt{n}$, where $\lambda=\sqrt{D_{0} \tau_{0}}$ is the length of one diffusion jump, which is usually of roughly atomic size or approximately coincides with the metal's crystal lattice period. It is known that rhenium with $1,373 \AA$ atomic radius and Re 1 $+1.33 \AA$, Re7 + $0.56 \AA$ ionic radii crystallizes in a hexagonal close-packed lattice ( $a=2.760 \AA, c=0.458 \AA$. Suppose that $\tau_{\text {diff }}=\tau_{0} \exp E_{\text {diff }} / k T$. Let's compute the diffusion length for three different exposure times $t_{1}=$ $10 \mathrm{~min}=600 \mathrm{~s}, t_{2}=2 \mathrm{~h}=7200 \mathrm{~s}$ and $t_{3}=10 \mathrm{~h}=36,000 \mathrm{~s}$ at several temperatures of rhenium, i.e., the average distance in angstroms, for which the Cs atoms (atomic radius of cesium is $2.67 \AA$, radius of the monovalent ion is $1.67 \AA$ ) shift deep into rhenium and the average lifetime of Cs atoms (Table 1) in the crystal lattice of rhenium. Comparison of the obtained results showed that the average displacements of diffusion for cesium atoms in $\operatorname{Re}(10 \overline{10})$ is less than in $\operatorname{Ir}(\mathrm{III})$.

It can be seen that even at a sufficiently high temperature of $T=2200 \mathrm{~K}$ and longer exposure times of $t=10 \mathrm{~h}$ cesium penetrates rhenium tape by a small distance of $480 \AA$, which makes $\sim 175$ atomic layers of rhenium, while $30 \mathrm{mkm}$ thick rhenium tape contains $\sim 10^{5}$ atomic layers. Consequently, almost all diffused cesium is probably located in a thin surface layer of rhenium. It can be said with a high probability that the above differences in the TDS spectra are associated with the rhenium and iridium crystal lattice structure, as well as the dimensions of atoms. The mentioned experimental data show that doping of iridium, rhenium with $\mathrm{K}, \mathrm{Cs}, \mathrm{Na}, \mathrm{Ba}$, $\mathrm{Sm}$, In, etc. atoms may lead to the change of 5d-zone of transition metals' electron density.

Dissolution of surface $C$ s in rhenium. Activation energy of $E$ diffusions of surface $C s$ in rhenium was found in special experiments. For this purpose, a two-dimensional layer of cesium intercalated with an initial concentration of $N$ was formed under the graphite monolayer on rhenium, then $T$ was increased and reduction of this concentration of $N$ was monitored (by flash) through diffusion of cesium in rhenium. It is obvious that the

\begin{tabular}{|c|c|c|c|c|}
\hline $\mathrm{T}, \mathrm{K}$ & $\tau_{\text {diff }}, \mathrm{s}$ & $L_{1}(\AA)$, for $t_{1}=600 \mathrm{~s}$ & $L_{2},(\AA)$ for $t_{1}=7200 \mathrm{~s}$ & $L_{3},(\AA)$ for $t_{1}=36,000 \mathrm{~s}$ \\
\hline 1600 & 100,730 & 0.2 & 0.7 & 1.7 \\
\hline 1700 & 8793 & 0.72 & 2.5 & 5.6 \\
\hline 1800 & 1007 & 2.2 & 7.9 & 16.5 \\
\hline 1900 & 145 & 5.6 & 19.4 & 43.5 \\
\hline 2000 & 25 & 7.2 & 46.8 & 104.7 \\
\hline 2100 & 5 & 30 & 104 & 234 \\
\hline 2200 & 1,2 & 62 & 214 & 478 \\
\hline
\end{tabular}


reduction of $\mathrm{d} N$ concentration of surface Cs during the time $\mathrm{d} t$ is associated with diffusion flow $v$ and average lifetime of adsorbed atoms Cs with respect to dissolution of $t_{p 1}=t_{0} \exp E_{p 1} /(k T)$ in rhenium with ratio

$$
\mathrm{d} N=-v \mathrm{~d} t=-N \mathrm{~d} t / t_{p 1}
$$

Integration of the relationship (12) gives us

$$
N=N \exp \left(-t / t_{\mathrm{m} 1}\right),
$$

where from we can deduce the activation energy of surface Cs dissolution in rhenium:

$$
E_{p 1}=\kappa T \ln \left(t / t_{0} \ln \left(N_{0} / N\right)\right)
$$

It appears that $E_{1 p}=5.65 \pm 0.05 \mathrm{eV}$.

Discharge of Cs from the bulk of rhenium to its surface. Let's consider diffusion of Cs from the bulk to the surface of rhenium. Let's say, $N_{1}$ is Cs concentration in the plane lagging behind the surface by the distance of a single diffusion jump. Then the flow of Cs coming to the surface and associated with average lifetime of atom with respect to the diffusion on surface $t_{p 1}=t_{0} \exp E_{p 1} /(k T)$, will equal to

$$
v=N_{1} / t_{1 p}=N_{1} / t_{0} \exp \left[E_{1 p} /(k T)\right] .
$$

We will register the flow of Cs atoms coming to metal surface by I ions of $\mathrm{Cs}^{+}$. From the condition of conserving the $v_{1 n}=v_{\partial e c}$ flow at $100 \%$ efficiency of ionization and $\mathrm{S}$ ion collection area, we get

$$
I=e v_{\text {des }} S=e N_{1} S / t_{0} \exp \left[E_{1 p} /(k T)\right] .
$$

Suppose that during the experiment, the $v_{1 n} t_{\text {exsp. }} \ll N_{1}$ correlation takes place. Then the flow $v_{1 n}$ will be constant and

$$
I=B \exp \left[-E_{1 p} /(k T)\right] .
$$

Experimentally obtained dependence $\ln I=f(1 / T)$ was linear and the activation energy of diffusion of Cs atoms of rhenium was found to be $E_{p 1}=5.85 \pm 0.05 \mathrm{eV}$. As can be seen from this correlation $E_{1 p}<E_{p 1}$, potential barrier for the diffusion of cesium atoms in the case of rhenium is also asymmetric, but the difference between energies of diffusion in Re and from Re was smaller than in the case of cesium atoms diffusion in Ir. In the simplest case, when the diffusion coefficient does not depend on the concentration of diffusible component, activation energy $E_{a}$ can be deduced from the slope of $\lg D$ dependence on 5040/T according to the Equation obtained by taking the logarithm of correlation (4). The results of these calculations will be given in the following materials.

\section{The Effectiveness of Diffusion and Influence of the Electric Field on Intercalation and Diffusion}

The proportion of flux or efficiency of $\delta$ diffusion diffusing in Re at irradiation of $\operatorname{Re}(1010)$-C with Cs flow was identified by two independent methods:

1) By surface ionization (PI) of cesium atoms in Re via the formula

$$
\delta(T)=1-\frac{I(T)}{I_{0}}
$$

where $I_{0}$ and $I(T)$ are cesium ion currents from a clean surface of rhenium with graphite monolayer respectively.

2) From comparison of the number of incident atoms of cesium on Re-C at this temperature for a certain time $t$ to the number of diffusing cesium atoms in rhenium during this time $\delta=\frac{v_{\text {diff. }}}{v_{\text {diff. }}+v_{\text {des. }}}$. Both methods gave almost identical results. Under the conditions $v=5 \times 10^{13} \mathrm{sm}^{-2} \cdot \mathrm{s}^{-1}, U=+2000 \mathrm{~V}, T=1700 \mathrm{~K}$, the effectiveness 
of cesium diffusion $\delta_{\mathrm{Cs}}$ in Re (1010)-C was determined via the above methods, which gave $\delta_{\mathrm{Cs}}=0.45$. This value $\delta_{\mathrm{Cs}}$ is more than $\delta_{\mathrm{Cs}}$ of cesium in the system $\operatorname{Ir}(\mathrm{III})-\mathrm{C}\left(\delta_{\mathrm{Cs}}=0.25\right)$. Thus, the formation of graphite monolayer on the surface of rhenium due to the huge increase in the lifetime of adsorbed cesium atoms under graphite islets as compared with those of rhenium with an open surface enormously increases the efficiency of surface diffusion of cesium in rhenium, which grows by about $\sim 10^{8}$ times from $1.5 \times 10^{-9}$ to 0.45 , approaching one. The effect of electric field on the intercalation and diffusion of cesium in Re-C similar to the one detected for potassium in Re-C [7] was observed. The intercalation and diffusion of cesium, just like that of potassium, occurs only in case of a positive potential of iridium tape. The noticeable intercalation and diffusion of cesium atoms started at $U \geq+150 \mathrm{~V}$ and increased with growth of voltage up to $4000 \mathrm{~V}$ roughly by linear law. At the potential of $600 \mathrm{~V}-700 \mathrm{~V}$, the intercalated phase reaches saturation and practically does not rise with growing voltage. The density of diffusion phase increases linearly with growth of $\mathrm{U}$ up to $4000 \mathrm{~V}$ (maximum voltage of power supply source). This effect is explained by decoration of the edges of graphite islets with $\mathrm{K}$ or Cs ions. At pre-irradiation of $\operatorname{Re}(1010)-C$ heated to high-temperature by the large flow $\left(10^{13}-10^{14} \mathrm{~cm}^{-2} \cdot \mathrm{s}^{-1}\right)$ of cesium atoms, a significant number (hundreds of monolayers) of cesium atoms diffused into rhenium during a long time (an hour, 2 hours, etc.). This followed from the analysis of TDS and also from measuring of PI current flow of cesium atoms diffusing from the volume at high temperatures $T=2200 \mathrm{~K}$. Therefore it is proposed to use rhenium as an effective source of cesium atoms and ions. For example, after exposure rhenium ribbon heated up to $T=1700 \mathrm{~K} \mathrm{Re}(1010)$-C in a stream of Cs atoms with density $v=1 \times 10^{14} \mathrm{~cm}^{-2} \cdot \mathrm{s}^{-1}$ for a time of $t=1 \mathrm{hr}$ at $T=$ $2200 \mathrm{~K}$ emitted a steady stream of $\mathrm{Cs}^{+}$ions with density $v=1 \times 10^{12} \mathrm{~cm}^{-2} \cdot \mathrm{s}^{-1}$ for the duration of $t=30 \mathrm{~min}$ and at $T=2150 \mathrm{~K}$, rhenium ribbon emitted steady stream of $\mathrm{Cs}^{+}$ions with density $v=5 \times 10^{11} \mathrm{~cm}^{-2} \cdot \mathrm{s}^{-1}$ for the duration of $t>1$ hour. Prolonged contact with the atmosphere of rhenium ribbon saturated with cesium had no effect on the reemitting of cesium from rhenium and iridium [10] tape again under high vacuum at high temperatures. This fact of prolonged existence of cesium within the volume of highly heated metal screen can be used in practice and requires development of a process model.

\section{References}

[1] Tontegode, A.Y. (1991) Progress in Surface Science, 38, 201429.

[2] Tontegode, A.Y. and Rutkov, E.V. (1993) Progress of Physical Sciences (Russian), 163, 1053.

[3] Tontegode, A.Y. and Yusifov, F.K. (1993) FTT, 35, 987-993.

[4] Tontegode, A.Y. and Yusifov, F.K. (1994) Surface, 4, 20-24.

[5] Orudjov, A.K, Dashdamirova, A.O. and Yolchiyeva, A.K. (2010) Physics of Metals and General Metallurgy, 109, 366-368.

[6] Orudjov, A.K. and Dashdamirov, A.O. (2007) Diffusion of Na Atoms in Ir with Irradiation of Ir-C Flux of Na Atoms. VII International Scientific Conference "Solid Body Chemistry and Modern Micro- and Nano-Systems", Kislovodsk, 17- 22 September 2007, 95-96.

[7] Orudjov, A.K. (2013) Physics of Metals and General Metallurgy, 114, 63-67.

[8] Orudjov, A.K., Nurullayev, Y.G. and Dashdamirov, A.O. (2005) News of Baku University, Series of Physical and Mathematical Sciences, 3, 140-149.

[9] Bockstein, B.S. and Yaroslavtsev, A.B. (2005) Diffusion of Atoms and Ions in Solids. M: MISA, 362.

[10] Orudjov, A.K. (2011) Physics of Metals and General Metallurgy, 111, 626-627. 